\title{
Electroweak baryogenesis at high bubble wall velocities
}

\author{
James M. Cline ${ }^{*}$ \\ Department of Physics, McGill University, 3600 Rue University, Montréal, Québec, Canada H3A 2T8
}

\author{
Kimmo Kainulainen $\oplus^{\dagger}$ \\ Department of Physics, P.O.Box 35 (YFL), FIN-40014 University of Jyväskylä, Finland; \\ Helsinki Institute of Physics, P.O. Box 64, FIN-00014 University of Helsinki, Finland; \\ and Theoretical Physics Department, CERN, 1211 Geneva 23, Switzerland
}

(Received 6 January 2020; accepted 9 March 2020; published 20 March 2020)

\begin{abstract}
It is widely believed that electroweak baryogenesis should be suppressed in strong phase transitions with fast-moving bubble walls, but this effect has never been quantitatively studied. We rederive fluid equations describing transport of particle asymmetries near the bubble wall without making the small-wall-velocity approximation. We show that the suppression of the baryon asymmetry is a smooth function of the wall speed and that there is no special behavior when crossing the sound speed barrier. Electroweak baryogenesis can thus be efficient also with strong detonations, generically associated with models with observably large gravitational waves. We also make a systematic and critical comparison of our improved transport equations to another one commonly used in the literature, based on the vacuum expectation value (VEV)-insertion formalism.
\end{abstract}

DOI: 10.1103/PhysRevD.101.063525

\section{INTRODUCTION}

Electroweak symmetry exhibits a phase transition in the early universe that is known to be a smooth crossover in the standard model (SM) 16 [1], but could become first order if new physics beyond the SM couples significantly to the Higgs boson. A strongly first order electroweak phase transition (EWPT) is one of the necessary requirements for electroweak baryogenesis (EWBG) [2-5], and it could also be a source of gravity waves that might be observed at LISA.

There is a perception that EWBG and observable gravity waves would tend to be mutually exclusive, however, since the latter require very strong phase transitions, which lead to fast-moving bubbles, with wall velocity $v_{w} \sim 1$. This makes it difficult for particle asymmetries to diffuse efficiently in front of the wall and bias sphalerons to create the baryon asymmetry. There may be some tension between the two effects, but until recently there have been few quantitative studies including transport of the particle asymmetries [6-11]. Most works have focused on the coexistence of observable gravitational waves with the

\footnotetext{
jcline@physics.mcgill.ca

kimmo.kainulainen@jyu.fi
}

Published by the American Physical Society under the terms of the Creative Commons Attribution 4.0 International license. Further distribution of this work must maintain attribution to the author(s) and the published article's title, journal citation, and DOI. Funded by SCOAP ${ }^{3}$. sphaleron washout condition $v / T \gtrsim 1$ [12-22], without taking into account the problem of reduced particle transport near the wall.

The theoretical deficit is in large part due to the fact that transport equations for the particle asymmetries have been derived using the approximation $v_{w} \ll 1$, making it impossible to reliably predict the baryon asymmetry at large $v_{w}$. Since it was believed that $v_{w} \lesssim 0.1$ in the SM [23-25] and the minimal supersymmetric standard model (MSSM) [26], the small- $v_{w}$ approximation seemed adequate at the time. In recent years, however, there has been increased interest in two-step phase transitions involving a scalar singlet field [27], which is able to generate stronger phase transitions with typically higher $v_{w}$. Such transitions can more easily satisfy the sphaleron washout constraint, and in addition can be a strong source of gravitational waves. It is therefore timely to revisit the transport equations relevant for EWBG and try to extend their applicability to higher $v_{w}$. We will show in particular that nothing special happens when the wall speed crosses the sound barrier, and that the baryon asymmetry only vanishes smoothly in the extreme limit $v_{w} \rightarrow 1 .^{1}$

This work has two main goals. The first is to update the fluid equations for the semiclassical force mechanism

\footnotetext{
${ }^{1}$ Throughout this work, $v_{w}$ is taken as a proxy for the relative speed between the bubble wall and the plasma in front of it. In realistic solutions of the fluid equations near the wall, it can happen that this relative velocity, which is the relevant quantity for diffusive transport, differs from the wall velocity as measured with respect to the plasma at infinite distance.
} 
[28-33] to arbitrary wall velocities. This is strongly motivated because the currently existing formulation [34] breaks down for wall velocities exceeding the sound speed. Our second purpose is to perform a quantitative comparison between the semiclassical method and the competing "VEV-insertion" approximation [35,36]. These two approaches agree that the particle densities contributing to the baryon asymmetry are determined by (quantum) Boltzmann equations, but it remains controversial what precise form they should take.

The semiclassical method is designed to be valid when the de Broglie wavelength of the particles, of order the inverse temperature $T^{-1}$, is smaller than the typical width of a bubble wall $L_{w}$. The interactions of particles with the wall can then be treated as coming from a semiclassical force that can be derived using the Wentzel-KramersBrillouin (WKB) approximation [29,37,38], or from the closed-time-path (CTP) formalism of the thermal field theory [30-33,39-42]. In the semiclassical approach the $C P$-violating force appears at the level of the Boltzmann equations. It is straightforward to approximate them by a set of moment equations with source terms induced by the force, which can be determined systematically in an expansion in powers of $\left(L_{w} T\right)^{-1}$ (though the subleading corrections have not been computed).

The VEV-insertion method is also derived starting from the CTP formalism. Here quantum Boltzmann equations are manipulated to yield their classical counterparts at the level of integrated particle densities. In this approach the source term is not easy to extract and one must make a rather drastic approximation, expanding a two-point function to leading order in the spatially varying Higgs field VEV $v(z)[35,36]$. This is known as the VEV-insertion approximation. It can be regarded as an expansion in powers of $v(z) / T$, which cannot be very small inside the bubble if the phase transition is sufficiently strong to avoid washout. It is hoped that since $v(z)$ is somewhat smaller inside the bubble wall, this can still be a reasonable approximation. But if that is the case, it must be capturing quite different physics from the WKB approach, since the two formalisms cannot be obviously reconciled, and in general they make quite different predictions.

For example, EWBG in the MSSM was analyzed using both formalisms [29,37,43-45], with the VEV-insertion method giving significantly larger estimates for the asymmetry. However, a systematic study of the differences between the two methods is lacking in the literature, in particular in comparing their predictions as a function of parameters characterizing the bubble wall. We will provide such a comparison in this work, for a prototypical model of $C P$ violation in the wall. As was the case for studies of EWBG in the MSSM, we will demonstrate a large discrepancy between the predictions of the two methods.
We start in Sec. II by arguing that the transport equations should not suffer from any sort of critical behavior for bubble walls that move near the speed of sound, but should rather only do so as $v_{w} \rightarrow 1$. In Sec. III we review the derivation of the WKB transport equations and the origin of their $v_{w}$ dependence. We point out an inconsistency in the approximations used in Ref. [34] (hereafter denoted FH06), and remedy it by a more careful evaluation of the coefficient functions for general values of $v_{w}$. In Sec. IV we introduce our fiducial model, and in Sec. V we compare the predictions of the FH06 equations and our improved fluid equations in the semiclassical approach. In Sec. VI we quantitatively compare the semiclassical approach to the VEV-insertion framework. Conclusions are given in Sec. VII.

\section{RELEVANCE OF WALL VELOCITY}

The basic idea for reducing the full Boltzmann equations to a set of coupled first order fluid equations for the chemical potential and velocity perturbation, in the context of electroweak baryogenesis, was set out in Ref. [28]. The method was elaborated for the MSSM in Ref. [29] and for general two-Higgs doublet models in Ref. [34]. In principle, one can always "integrate out" the velocity perturbation and convert the coupled system into a single second order diffusion equation for the chemical potentials, as was done in Ref. [29]. However, this is complicated by the fact that any particle that couples strongly to the Higgs boson (as required to source electroweak baryogenesis) has a mass that varies within the bubble wall, and therefore the coefficients in the diffusion equation are functions of $z$, the distance transverse to the wall. If one makes the crude approximation of ignoring baryon violation by sphalerons inside the bubble and taking the masses to vanish outside, then the $z$-dependence goes away and Green's function techniques can be used to solve the diffusion equations. However, this effectively approximates the wall as being very thin, which is inconsistent with the semiclassical expansion underlying the whole fluid approach. For quantitative results, one should numerically solve the coupled equations keeping track of the full $z$-dependence of the coefficients.

An important contribution of FH06 was to calculate all of the coefficient functions $K_{i}^{\mathrm{FH}}(x)$, where $x=m(z) / T$, appearing in the fluid equations. In their approach, these functions are independent of the wall velocity $v_{w}$, which was achieved by expanding to leading order in $v_{w}$. Therefore one could question to what extent this formalism can be accurate for walls with large wall velocities.

It is expected that diffusion lengths should diminish as $v_{w}$ gets large. One can make a simple estimate to quantify this statement, by asking what fraction $F$ of particles in the plasma are moving faster than the wall, in its direction of motion. We would expect that only of order is this fraction able to contribute to the diffusion 


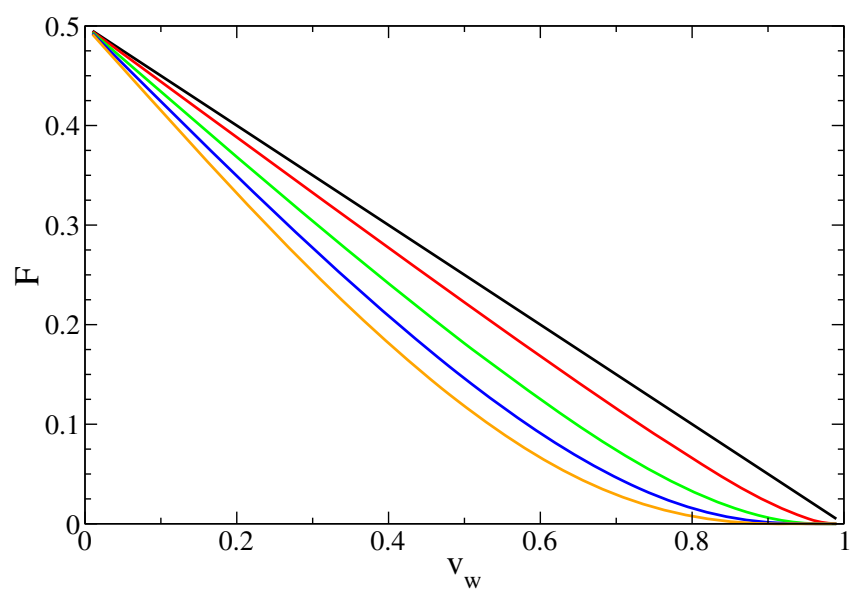

FIG. 1. Fraction of plasma particles that can stay ahead of a bubble wall moving at speed $v_{w}$. Different curves are for fermions with $m / T=0,1,2,3,4$ (top to bottom).

tail for the $C P$ asymmetry in front of the wall. It is straightforward to show ${ }^{2}$ that

$$
\mathrm{F}=\frac{\frac{1}{2} \int_{\gamma_{w} v_{w} m}^{\infty} \mathrm{d} p p^{2}\left(1-v_{w} E / p\right) /\left(e^{\beta E}+1\right)}{\int_{0}^{\infty} \mathrm{d} p p^{2} /\left(e^{\beta E}+1\right)}
$$

for a massive fermion. In Fig. 1 we plot $\mathrm{F}$ versus $v_{w}$ for particles with increasing values of $m / T$. It is clear that nothing dramatic happens near the sound speed $v_{s} \cong 1 / \sqrt{3}$; instead $v_{w}=1$ is the only critical speed.

\section{DERIVATION OF TRANSPORT EQUATIONS}

The Boltzmann equation acting on the unperturbed distribution functions can be written in the wall frame as

$$
\left(v_{g} \partial_{z}+F \partial_{p_{z}}\right) f=\mathcal{C}[f] .
$$

For a fermion with a $C P$-violating complex mass term $\hat{m}(z)=m(z) e^{i \gamma^{5} \theta(z)}[29,31]$,

$$
\begin{gathered}
v_{g}=\frac{p_{z}}{E_{w}} \\
F=-\frac{\left(m^{2}\right)^{\prime}}{2 E_{w}}+s s_{k_{0}} \frac{\left(m^{2} \theta^{\prime}\right)^{\prime}}{2 E_{w} E_{w z}}
\end{gathered}
$$

where ' denotes $\partial_{z}$. Here $E_{w z}^{2}=E_{w}^{2}-\mathbf{p}_{\|}^{2}$ and $E_{w}$ is the conserved wall frame energy. $s_{k_{0}}=1$ for particles and -1 for antiparticles, and $s= \pm 1$ for the states that are the eigenstates of the spin $s$ in the $z$-direction in the frame where the momentum of the state parallel to the wall $\mathbf{p}_{\|}$

\footnotetext{
${ }^{2}$ By first doing the angular integral over $\cos \theta=p_{z} / p$.
}

vanishes. For the wall frame helicity eigenstates one should replace $[46]^{3}$

$$
s \rightarrow s_{h}=h \gamma_{\|} \frac{p_{z}}{|\mathbf{p}|} \equiv h s_{\mathrm{p}},
$$

where $h= \pm 1$ is the helicity and $\gamma_{\|}=E_{w} / E_{w z}$ is the Lorentz boost for going to the frame where $\mathbf{p}_{\|}=0$. In practice the difference between the two spin bases is small [46]. In particular in the massless limit $s_{h}=h \operatorname{sign}\left(p_{z}\right)$. Equation (3) is actually the definition of the physical momentum $p_{z}$ from the group velocity determined by the WKB dispersion relation [29,31]. It is convenient to write this relation in a form that defines $E_{w}$ in terms of the physical momentum:

$$
E_{w} \approx E-s_{h} s_{k_{0}} \frac{m^{2} \theta^{\prime}}{2 E E_{z}} \equiv E+s_{h} s_{k_{0}} \Delta E,
$$

where $E \equiv \sqrt{\mathbf{p}^{2}+m^{2}}$ and $E_{z} \equiv \sqrt{p_{z}^{2}+m^{2}}$. Using these variables, Eqs. (3) and (4) become

$$
\begin{gathered}
v_{g}=\frac{p_{z}}{E}+s_{h} s_{k_{0}} \frac{m^{2} \theta^{\prime}}{2 E^{2} E_{z}} \\
F=-\frac{\left(m^{2}\right)^{\prime}}{2 E}+s_{h} s_{k_{0}}\left(\frac{\left(m^{2} \theta^{\prime}\right)^{\prime}}{2 E E_{z}}-\frac{m^{2}\left(m^{2}\right)^{\prime} \theta^{\prime}}{4 E^{3} E_{z}}\right) .
\end{gathered}
$$

Equations (6)-(8) agree with those derived in [34] when one sets $s_{h} \rightarrow s$.

For bosons there is no $\mathrm{CP}$-violating semiclassical force at this order in the gradient expansion [29]. However, the $C P$-even kinetic force remains, and so all equations are valid for bosonic degrees of freedom if one simply sets $s_{k_{0}}=0$ everywhere.

The starting point for deriving fluid equations from the Boltzmann equation is to expand particle distribution functions around the equilibrium distribution. Because the kinetic momentum $p_{z}$ is conserved in collisions, the expansion in the rest frame of the bubble wall looks like

$$
f=\frac{1}{e^{\beta\left[\gamma_{w}\left(E_{w}+v_{w} p_{z}\right)-\mu\right]} \pm 1}+\delta f
$$

where $\gamma_{w}=1 / \sqrt{1-v_{w}^{2}}$. Here $\mu$ is a pseudochemical potential that defines the particle asymmetry and $\delta f$ is an extra term whose specific form should be left unspecified, ${ }^{4}$ except for stipulating that

\footnotetext{
${ }^{3}$ We correct a typo in Eq. (E3) of [46], by replacing $\left|p_{z}\right| \rightarrow p_{z}$.

${ }^{4}$ Unlike the perturbation in the chemical potential, whose algebraic form is enforced by fast elastic scattering processes, the form of the velocity perturbation is not predictable [29]. Thus, assuming a specific ansatz for its shape in momentum space can lead to unphysical behavior, in particular at large $v_{w}$.
} 


$$
\int \mathrm{d}^{3} p \delta f=0
$$

This condition is just the definition of $\mu$; it ensures that $\delta f$ does not affect the local particle density.

\section{A. Classification by $C P$ parity}

Next observe that the semiclassical force in (8) contains two distinct pieces: the first, the $C P$-even term, is equal for particles and antiparticles while the second, the $C P$-odd term, is opposite for particles and antiparticles. The $C P$-even term is of first order in gradients while the $C P$-odd term is of second order. Because of this hierarchy, one can solve the $C P$-even and $C P$-odd equations separately. To this end we introduce the definitions

$$
\begin{gathered}
\mu \equiv \mu_{e}+s_{k_{0}} \mu_{o}, \\
\delta f \equiv \delta f_{e}+s_{k_{0}} \delta f_{o} .
\end{gathered}
$$

Using these together with Eq. (6) we can write Eq. (9) as

$$
f \approx f_{0 w}+\Delta f_{e}+s_{k_{0}} \Delta f_{o}
$$

where, expanding to leading consistent order in both $C P$ even and $C P$-odd quantities,

$$
\begin{aligned}
\Delta f_{e} & =-\mu_{e} f_{0 w}^{\prime}+\delta f_{e}, \\
\Delta f_{o} & =\left(-\mu_{o}+s_{h} \gamma_{w} \Delta E\right) f_{0 w}^{\prime}-s_{h} \gamma_{w} \Delta E f_{0 w}^{\prime \prime} \mu_{e}+\delta f_{o},
\end{aligned}
$$

where the prime denotes $\mathrm{d} / \mathrm{d}\left(\gamma_{w} E\right)$ and

$$
f_{0 w}=\frac{1}{e^{\beta\left[\gamma_{w}\left(E_{w}+v_{w} p_{z}\right)\right]} \pm 1} .
$$

The expansion (12) is also necessary for bosons. Even though bosonic equations do not have direct $C P$-violating sources at the order to which we are working, they can inherit $C P$-violating perturbations from their interactions with fermions.

To derive the $C P$-even equation we drop the $C P$-odd parts proportional to $s_{k_{0}}$ in the expansion (12) and in Eqs. (7) and (8) for the group velocity and the semiclassical force. After this the Boltzmann equation (2) immediately becomes

$$
L\left[\mu_{e}, \delta f_{e}\right]=\mathcal{S}_{e}+\delta \mathcal{C}_{e},
$$

where the Liouville operator is defined as

$$
\begin{aligned}
L[\mu, \delta f] \equiv & -\frac{p_{z}}{E} f_{0 w}^{\prime} \partial_{z} \mu+v_{w} \gamma_{w} \frac{\left(m^{2}\right)^{\prime}}{2 E} f_{0 w}^{\prime \prime} \mu \\
& +\frac{p_{z}}{E} \partial_{z} \delta f-\frac{\left(m^{2}\right)^{\prime}}{2 E} \partial_{p_{z}} \delta f,
\end{aligned}
$$

and the $C P$-even source term is

$$
\mathcal{S}_{e}=v_{w} \gamma_{w} \frac{\left(m^{2}\right)^{\prime}}{2 E} f_{0 w}^{\prime}
$$

The collision term for the $C P$-even perturbation $\delta \mathcal{C}_{e}$ is model dependent, and we do not specify it further until Sec. IV. The $C P$-even equations (15)-(17) are valid for both bosons and fermions, and are helicity independent, unlike their $C P$-odd counterparts.

\section{B. $C P$-odd equation}

In the $C P$-odd sector we must account for the helicity. Because the relevant physical quantity for EWBG is the left-handed chiral asymmetry in front of the wall, one often concentrates only on the negative helicity sector, ${ }^{5}$ but to be general we keep the full helicity dependence. Projecting out the $C P$-odd part of the Boltzmann equation (2) requires some work, but the final result is analogous to Eq. (15) up to source and collision terms:

$$
L\left[\mu_{o}, \delta f_{o}\right]=\mathcal{S}_{o}+\delta \mathcal{C}_{o},
$$

where the $C P$-odd source term is

$$
\begin{aligned}
\mathcal{S}_{o h}= & -v_{w} \gamma_{w} h s_{\mathrm{p}} \frac{\left(m^{2} \theta^{\prime}\right)^{\prime}}{2 E E_{z}} f_{v_{w}}^{\prime} \\
& +v_{w} \gamma_{w} h s_{p} \frac{m^{2}\left(m^{2}\right)^{\prime} \theta^{\prime}}{4 E^{2} E_{z}}\left(\frac{f_{v_{w}}^{\prime}}{E}-\gamma_{w} f_{v_{w}}^{\prime \prime}\right)
\end{aligned}
$$

and the collision integral $\delta C_{o}$ is again model dependent, which we will specify later.

Setting $h=-1$ and $s_{\mathrm{p}}=\operatorname{sign}\left(p_{z}\right)$, Eq. (19) agrees with FH06 up to an overall sign. A number of $C P$-odd source terms computed in FH06, proportional to $\mu_{e}$ and $\delta f_{e}$, were dropped during evaluation, since they are higher order in gradients.

\section{Moment expansion}

One could solve $\mu_{e, o}$ and $\delta f_{e, o}$ directly from Eqs. (15) and (18). It is more economical, however, to first reduce them to a set of moment equations. Because of their identical forms, the equations for both $C P$ parities can be treated simultaneously. We introduce moments by integrating over $p$, being weighted by $\left(p_{z} / E\right)^{l}$, and dividing by a normalization factor

\footnotetext{
${ }^{5}$ This is reasonable when masses vanish in front of the wall. If this is not the case, one should compute the asymmetry in the positive helicity sector as well, and project out the left chiral asymmetry from both helicity contributions. Note that while the Liouville terms are identical for both helicities, the sources are equal and opposite. The collision terms are also helicity dependent.
} 


$$
\begin{aligned}
N_{1} & \equiv \int \mathrm{d}^{3} p f_{0 w, \mathrm{FD}}^{\prime}=\gamma_{w} \int \mathrm{d}^{3} p f_{0, \mathrm{FD}}^{\prime} \\
& \equiv \gamma_{w} \hat{N}_{1}=-\gamma_{w} \frac{2 \pi^{3}}{3} T^{2}
\end{aligned}
$$

where $f_{0, \mathrm{FD}}$ is the equilibrium distribution function for a massless fermion in the fluid frame. It is convenient to normalize even the equations for a massive particle using this universal factor, so that when several species of particles are coupled through their interactions, the rates in the collision terms are related in a simple way between equations for different species. Then terms appearing in the fluid equations can be expressed as averages over the phase space of the form

$$
\langle X\rangle \equiv \frac{1}{N_{1}} \int \mathrm{d}^{3} p X
$$

In particular, the integrals over $\delta f$ define the velocity perturbations

$$
u_{\ell} \equiv\left\langle\left(\frac{p_{z}}{E}\right)^{\ell} \delta f\right\rangle
$$

The $\ell$ th moment of the evolution equation can then be written as

$$
\left\langle\left(\frac{p_{z}}{E}\right)^{\ell} L\right\rangle=\left\langle\left(\frac{p_{z}}{E}\right)^{\ell}(\mathcal{S}+\delta \mathcal{C})\right\rangle .
$$

Next we focus on the Liouville term (16), which contains important $v_{w}$ dependence.

\section{Liouville term}

Our goal is to reduce the system (23) to a closed set of equations for $\mu$ 's and the velocity perturbations (22). We include only the two lowest moments, as has been done so far in the literature $[34,46,47] .{ }^{6}$ Taking the zeroth and first moment of the Liouville operator we find

$$
\begin{aligned}
\langle L\rangle= & -D_{1} \mu^{\prime}+u_{1}^{\prime}+v_{w} \gamma_{w}\left(m^{2}\right)^{\prime} Q_{1} \mu \\
\left\langle\frac{p_{z}}{E} L\right\rangle= & -D_{2} \mu^{\prime}+u_{2}^{\prime}+v_{w} \gamma_{w}\left(m^{2}\right)^{\prime} Q_{2} \mu \\
& +\left(m^{2}\right)^{\prime}\left\langle\frac{1}{2 E^{2}} \delta f\right\rangle
\end{aligned}
$$

where ' again denotes $\partial_{z}$ except when acting on the distribution functions, where it denotes $\partial_{\gamma_{w} E}$, and we introduce the functions

\footnotetext{
${ }^{6}$ Reference [48] also considered a temperature perturbation, but this is an ansatz for the distribution function, rather than a systematic expansion in velocity moments.
}

$$
\begin{gathered}
D_{\ell} \equiv\left\langle\left(\frac{p_{z}}{E}\right)^{\ell} f_{0 w}^{\prime}\right\rangle, \\
Q_{\ell} \equiv\left\langle\left(\frac{p_{z}^{\ell-1}}{2 E^{\ell}}\right) f_{0 w}^{\prime \prime}\right\rangle .
\end{gathered}
$$

The $D$ - and $Q$-functions are defined separately for bosons and for fermions, since the distribution function $f_{0 w}$ differs in the two cases. In the small $v_{w}$-limit they reduce to the FH06-functions as $D_{1} \rightarrow-v_{w} K_{1}^{\mathrm{FH}}, D_{2} \rightarrow K_{4}^{\mathrm{FH}}$, and $Q_{1} \rightarrow K_{2}^{\mathrm{FH}}$. The $Q_{2}$ term was, however, overlooked in $\mathrm{FH} 06$.

Equation (25) contains two problematic terms: $u_{2}$ is higher order in the expansion than the order to which we are working, and the last term is not obviously related to velocity perturbations (22). To treat the first term we need to introduce a truncation scheme, which relates $u_{2}$ to $u_{1}$. (More generally one should relate the $n$th moment to moments $u_{\ell}$ with $\ell<n$.) Here we adopt a simple linear relation, henceforth denoting $u_{1}=u: u_{2} \equiv R u$, where $R$ is a function to be defined shortly.

To define the last term in (25) we need a further factorization assumption. Following Ref. [29] and FH06, for any $\mathcal{X}$ that does not correspond to a velocity perturbation we replace

$$
\begin{aligned}
& \langle\mathcal{X} \delta f\rangle \rightarrow\left[\mathcal{X}\left(E / p_{z}\right)\right] u, \\
& {[\mathcal{X}] \equiv \frac{1}{N_{0}} \int d^{3} p \mathcal{X} f_{0 w},}
\end{aligned}
$$

where $N_{0}$ is another normalization factor,

$$
N_{0}=\int d^{3} p f_{0 w}=\gamma_{w} \int d^{3} p f_{0} \equiv \gamma_{w} \hat{N}_{0}
$$

Unlike $N_{1}, N_{0}$ is defined in terms of the massive distribution function $f_{0}$ of the particle under consideration.

In Eq. (29), it may happen that $\mathcal{X}$ does not have any power of $p_{z}$ to be canceled by the factor $E / p_{z}$ in Eq. (28). Nevertheless the integral can be defined using the Cauchy principal value. In particular,

$$
\left\langle\frac{1}{2 E^{2}} \delta f\right\rangle \rightarrow\left[\frac{1}{2 p_{z} E}\right] u \equiv \bar{R} u .
$$

After performing the singular angular integral using the principal value prescription, we find

$$
\bar{R}=\frac{\pi}{\gamma_{w}^{2} \hat{N}_{0}} \int_{m}^{\infty} \mathrm{d} E \ln \left|\frac{p-v_{w} E}{p+v_{w} E}\right| f_{0} .
$$

This should reduce to $v_{w} \tilde{K}_{6}^{\mathrm{FH}}$ at leading order in $v_{w}$, but due to a mistake in the evaluation of $\tilde{K}_{6}^{\mathrm{FH}}$ in FH06 it does not. 
Following FH06 we use the factorization rule also to define the truncation scheme,

$$
u_{2}=\left\langle\left(\frac{p_{z}}{E}\right)^{2} \delta f\right\rangle \rightarrow\left[\frac{p_{z}}{E}\right] u \equiv R u,
$$

where the bracket average [.] is defined in (29). Then $R$ becomes just the expectation value of the fluid velocity in the wall frame,

$$
R=-v_{w},
$$

which is an exact result. Comparing with FH06 $R=$ $v_{w} \tilde{K}_{5}^{\mathrm{FH}}$, this implies that $\tilde{K}_{5}^{\mathrm{FH}}=-1$ exactly. Although unstated in FH06, it is indeed the case.

The factorization and the truncation rules are, of course, somewhat arbitrary. It is therefore reassuring that the $\bar{R}$-term has but a weak effect on solutions: toggling between the choice (32) versus setting $\bar{R} \equiv 0$ changes the final baryon asymmetry at the level of a few percent. Moreover the definition (34) for $R$ is reasonable because $-v_{w}$ is roughly the ratio between adjacent source terms, order by order in the moment expansion.

\section{E. Sources and collision terms}

Assembling the previous results, and including the collision and source terms, the fluid equations can be presented in full detail. Defining a vector $w=(\mu, u)^{T}$, the general form of the two moment equations may be expressed as

$$
A w^{\prime}+\left(m^{2}\right)^{\prime} B w=S+\delta C,
$$

where

$$
A=\left(\begin{array}{cc}
-D_{1} & 1 \\
-D_{2} & R
\end{array}\right), \quad B=\left(\begin{array}{cc}
v_{w} \gamma_{w} Q_{1} & 0 \\
v_{w} \gamma_{w} Q_{2} & \bar{R}
\end{array}\right),
$$

and $S=\left(S_{1}, S_{2}\right)^{T}$ with $S_{1}=\langle\mathcal{S}\rangle$ and $S_{2}=\left\langle\left(p_{z} / E\right) \mathcal{S}\right\rangle$, and similarly for the $\delta C$ vector. The form (36) is generic to both $C P$-even and $C P$-odd sectors, which are only distinguished by their respective source terms.

Let us consider the source terms first. In the $C P$-even sector one finds using (17)

$$
S_{\ell}^{e}=v_{w} \gamma_{w}\left(m^{2}\right)^{\prime} Q_{\ell}^{e}
$$

with the definition

$$
Q_{\ell}^{e} \equiv\left\langle\frac{p_{z}^{\ell-1}}{2 E^{\ell}} f_{0 w}^{\prime}\right\rangle
$$

In the small- $v_{w}$ limit one finds $Q_{2}^{e} \rightarrow K_{3}^{\mathrm{FH}}$.
In the $C P$-odd sector, using Eq. (19) similarly gives

$$
S_{h \ell}^{o}=-v_{w} \gamma_{w} h\left[\left(m^{2} \theta^{\prime}\right)^{\prime} Q_{\ell}^{8 o}-\left(m^{2}\right)^{\prime} m^{2} \theta^{\prime} Q_{\ell}^{9 o}\right],
$$

where the coefficient functions are

$$
\begin{gathered}
Q_{\ell}^{8 o} \equiv\left\langle\frac{s_{\mathrm{p}} p_{z}^{\ell-1}}{2 E^{\ell} E_{z}} f_{0 w}^{\prime}\right\rangle, \\
Q_{\ell}^{9 o} \equiv\left\langle\frac{s_{\mathrm{p}} p_{z}^{\ell-1}}{4 E^{\ell+1} E_{z}}\left(\frac{1}{E} f_{0 w}^{\prime}-\gamma_{w} f_{0 w}^{\prime \prime}\right)\right\rangle,
\end{gathered}
$$

with $s_{\mathrm{p}}$ defined in (5). Setting $s_{\mathrm{p}} \rightarrow \operatorname{sign}\left(p_{z}\right)$ one finds that $Q_{2}^{8 o} \rightarrow K_{8}^{\mathrm{FH}}$ and $Q_{2}^{9 o} \rightarrow K_{9}^{\mathrm{FH}}$ in the small $v_{w}$-limit. Moreover, in previous work, the approximation $S_{1}=0$ was always made, because it is $O\left(v_{w}^{2}\right)$. For large velocities there is no hierarchy between $S_{1}$ and $S_{2}$ and one must include both sources.

It remains to consider the collision integrals. Both phase space averages $\delta C_{1} \equiv\langle\delta \mathcal{C}\rangle$ and $\delta C_{2} \equiv\left\langle\left(p_{z} / E\right) \delta \mathcal{C}\right\rangle$ are normalized using $N_{1}^{-1}$, Eq. (20). The collision term moments are derived following Appendix A of Ref. [29],

$$
\begin{aligned}
& \delta C_{1}=K_{0} \sum_{i} \Gamma_{i} \sum_{j} s_{i j} \frac{\mu_{j}}{T}, \\
& \delta C_{2}=-\Gamma_{\mathrm{tot}} u-v_{w} \delta C_{1} .
\end{aligned}
$$

Here $s_{i j}=1(-1)$ if the corresponding species is in the initial (final) state in the interaction with rate $\Gamma_{i}$, and $\Gamma_{\text {tot }}=$ $\sum_{i} \Gamma_{i}$ is the total interaction rate, including elastic channels that do not contribute to the sum in $\langle\delta \mathcal{C}\rangle$. The normalization factor

$$
K_{0} \equiv-\left\langle f_{0 w}\right\rangle=-\frac{\hat{N}_{0}}{\hat{N}_{1}}
$$

was neglected in FH06. For a massless fermion, for example, $K_{0} \cong 1.1$, Eqs. (42) are valid for both the $C P$ even and the $C P$-odd cases.

Equations (35) obviously depend on a large number of coefficient functions: $D_{\ell}, Q_{\ell}, R, \bar{R}, Q_{\ell}^{e}, Q_{\ell}^{8 o}, Q_{\ell}^{9 o}$, and $K_{0}$. Most of these depend on the wall velocity $v_{w}$ and the dimensionless ratio $x=m / T$. However, they are universal and model independent. In practice, we compute them on a grid of $x$ and $v_{w}$ values and spline fit them. Explicit expressions are given for all the integrals defining them in the Appendix A.

\section{F. Critical speed predictions}

There is a widespread notion, apparently originating from Ref. [49], that diffusion is inefficient for wall speeds exceeding the plasma sound speed. This would mean in particular that EWBG would not be feasible for detonation walls, corresponding to very strong phase transitions, often 
invoked in the context of gravitational wave production. This assertion is not true, as we shall show, but it turns out that the FH06 equations are, quite fortuitously, consistent with the false assumption.

We noted that the fluid equations can be written in the matrix form $A w^{\prime}=F[w]$, where $A$ is given in Eq. (36), while in FH06 the $A$ matrix is

$$
A_{\mathrm{FH}}=\left(\begin{array}{cc}
v_{w} K_{1}^{\mathrm{FH}} & 1 \\
-K_{4}^{\mathrm{FH}} & -v_{w}
\end{array}\right),
$$

setting $\tilde{K}_{5}^{\mathrm{FH}}=-1$ as mentioned above. One can solve for the value of $v_{w}$ where $A$ becomes singular (noninvertible) using $\operatorname{det}(A)=0$. If a solution exists for $v_{w}<1$, it implies a critical speed $v_{c}$ beyond which diffusion is quenched. The exact prediction using the $A$-matrix in (36) gives (recalling that $R=-v_{w}$ )

$$
v_{c}=-\left(\frac{D_{2}}{D_{1}}\right)_{v_{w}=v_{c}} \Rightarrow v_{c}=1,
$$

whereas the approximate FH06-condition gives a different velocity

$$
v_{c}^{\prime}=\left|\frac{K_{4}^{\mathrm{FH}}}{K_{1}^{\mathrm{FH}}}\right|_{v_{w}=0}^{1 / 2} .
$$

The dependence of $v_{c}^{\prime}$ on $m / T$ as obtained in the FH06case (46) is shown in Fig. 2 for a Fermi-Dirac distribution. (The corresponding curves for bosons look similar.) For light particles the quench limit is maximal and very close to the sound speed, but this is a mere coincidence due to inappropriate use of the small $v_{w}$-approximation. Indeed, from (45), employing full $v_{w}$-dependent function, we find that $v_{c}=1$, in accordance with the arguments given in Sec. II. Thus diffusion efficiency should go to zero

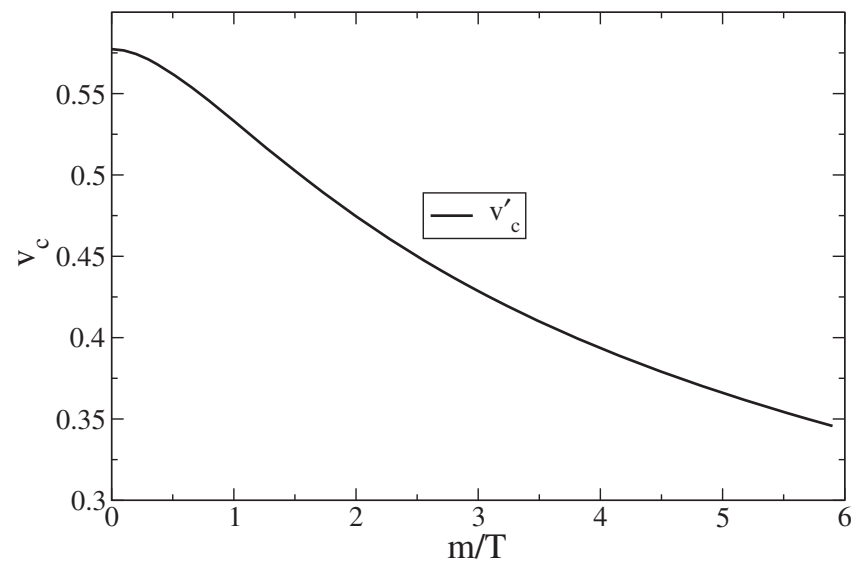

FIG. 2. Naive prediction for the critical wall velocity from FH06 equations, as a function of $m / T$. The correct value, using the full $v_{w}$-dependence of the $D_{\ell}$ functions, is $v_{c}=1$. smoothly as $v_{w} \rightarrow 1$, with no particular features at the sound speed, $v_{w}=v_{s}$. We will show that this indeed is the case.

\section{PHENOMENOLOGICAL MODEL}

To illustrate the consequences of our improved transport equations, we will compute the baryon asymmetry that they predict in a prototypical model that gives rise to EWBG, where the top quark mass has a $z$-dependent $C P$-violating phase in the bubble wall. The mass term can be written as

$$
m_{t}(z)\left(\bar{t}_{\mathrm{L}} e^{i \theta(z)} t_{\mathrm{R}}+\bar{t}_{\mathrm{R}} e^{-i \theta(z)} t_{\mathrm{L}}\right)
$$

in terms of the chiral components, where $m_{t}=y_{t} v(z)$ and $v(z)$ is the Higgs VEV that varies spatially within the wall. It can occur in two-Higgs-doublet models or in singletplus-doublet models where a dimension-5 operator such as $i(s / \Lambda) \bar{Q}_{3} H t_{\mathrm{R}}$ contributes a phase to the top mass, if $s$ also gets a VEV in the bubble wall. In such a model, the effective top quark mass term takes the form

$$
y_{t} h(z) \bar{t}_{\mathrm{L}}\left(1+i \frac{s(z)}{\Lambda}\right) t_{\mathrm{R}}+\text { H.c. }
$$

which implies

$$
\begin{aligned}
m_{t}(z) & =y_{t} h(z) \sqrt{1+s^{2}(z) / \Lambda^{2}}, \\
\theta(z) & =\tan ^{-1} \frac{s(z)}{\Lambda} .
\end{aligned}
$$

Here we will not consider the $C P$-even equations, which would be relevant for computing the wall speed and shape. Instead, we concentrate on the $C P$-odd sector and take a phenomenological approach, where $v_{w}$ is treated as a free parameter, and the VEVs $h(z)$ and $s(z)$ are modeled as

$$
\begin{aligned}
& h(z)=\frac{v_{n}}{2}\left(1-\tanh \frac{z}{L_{w}}\right), \\
& s(z)=\frac{w_{n}}{2}\left(1+\tanh \frac{\left(z-\delta_{w}\right)}{L_{s}}\right) .
\end{aligned}
$$

We are primarily interested in the $v_{w}$-dependence of the results and therefore choose fiducial values for the other parameters,

$$
\begin{aligned}
v_{n} & =\frac{1}{2} w_{n}=T_{n}, & \Lambda & =1 \mathrm{TeV}, \\
L_{w} & =L_{s}=\frac{5}{T_{n}}, & \delta_{w} & =0,
\end{aligned}
$$

in terms of the nucleation temperature, taken to be $T_{n}=100 \mathrm{GeV}$. 


\section{A. Fluid equation network}

With the tools and notation developed in the previous section one can express the complicated equation network in a compact form. Our system consists of four particle species $^{7}$ strongly coupled by the top-Yukawa interactions: left and right helicity tops are, respectively, denoted by $t_{\mathrm{L}}$ and $t_{\mathrm{R}}$, left helicity bottom by $b_{\mathrm{L}}$, and the complex Higgs particle by $h$. We neglect the small difference between helicity and chirality of the fermions here. There are eight dependent variables, combined into four 2-vectors $w_{i}=$ $\left(\mu_{o i}, u_{o i}\right)^{T}$ for $i=t_{\mathrm{L}}, b_{\mathrm{L}}, t_{\mathrm{R}}, h$, which obey

$$
\begin{aligned}
A_{t} w_{t_{\mathrm{L}}}^{\prime}+m_{t}^{2 \prime} B_{t} w_{t_{\mathrm{L}}}-\delta C_{t_{\mathrm{L}}} & =S_{t}, \\
A_{b} w_{b_{\mathrm{L}}}^{\prime}+m_{b}^{2 \prime} B_{b} w_{b_{L}}-\delta C_{b_{\mathrm{L}}} & =S_{b}, \\
A_{t} w_{t_{\mathrm{R}}}^{\prime}+m_{t}^{2 \prime} B_{t} w_{t_{\mathrm{R}}}-\delta C_{t_{\mathrm{R}}} & =-S_{t}, \\
A_{h} w_{h}^{\prime}+m_{h}^{2 \prime} B_{h} w_{h}-\delta C_{h} & =0,
\end{aligned}
$$

where the $A$ - and $B$-matrices are defined in (36) and the sources are $S_{i}=\left(S_{i 1}, S_{i 2}\right)^{T}$, $S_{i \ell}$ given by Eq. (39) taking $h \equiv-1$ for the left-handed fermions. In practice $S_{b}$ is negligible due to the smallness of the bottom Yukawa coupling.

In addition to top-Yukawa interactions, we account for the $W$ boson interactions that tend to equalize the $t_{\mathrm{L}}$ and $b_{\mathrm{L}}$ chemical potentials, the strong sphalerons, top mass insertions (helicity flips) that damp the combination $\mu_{t_{\mathrm{L}}}-\mu_{t_{\mathrm{R}}}$ $\left(\mu_{h}\right)$, and Higgs damping from electroweak symmetry breaking. These are the same collision terms as in FH06. Explicitly $\delta \mathcal{C}_{i} \equiv\left(K_{0}^{i} \delta \overline{\mathcal{C}}_{1}^{i}, \delta \mathcal{C}_{2}^{i}\right)^{T}$, where

$$
\begin{aligned}
\delta \overline{\mathcal{C}}_{1}^{t_{\mathrm{L}}}= & \Gamma_{y}\left(\mu_{t_{\mathrm{L}}}-\mu_{t_{\mathrm{R}}}+\mu_{h}\right)+\Gamma_{\mathrm{m}}\left(\mu_{t_{\mathrm{L}}}-\mu_{t_{\mathrm{R}}}\right) \\
& +\Gamma_{\mathrm{W}}\left(\mu_{t_{\mathrm{L}}}-\mu_{b_{\mathrm{L}}}\right)+\tilde{\Gamma}_{\mathrm{SS}}\left[\mu_{i}\right], \\
\delta \overline{\mathcal{C}}_{1}^{b_{\mathrm{L}}}= & \Gamma_{y}\left(\mu_{b_{\mathrm{L}}}-\mu_{t_{\mathrm{R}}}+\mu_{h}\right) \\
& +\Gamma_{\mathrm{W}}\left(\mu_{b_{\mathrm{L}}}-\mu_{t_{\mathrm{L}}}\right)+\tilde{\Gamma}_{\mathrm{SS}}\left[\mu_{i}\right], \\
\delta \overline{\mathcal{C}}_{1}^{t_{\mathrm{R}}}= & -\Gamma_{y}\left(\mu_{t_{\mathrm{L}}}+\mu_{b_{\mathrm{L}}}-2 \mu_{t_{\mathrm{R}}}+2 \mu_{h}\right) \\
& +\Gamma_{\mathrm{m}}\left(\mu_{t_{\mathrm{R}}}-\mu_{t_{\mathrm{L}}}\right)-\tilde{\Gamma}_{\mathrm{SS}}\left[\mu_{i}\right], \\
\delta \overline{\mathcal{C}}_{1}^{h}= & \tilde{\Gamma}_{y}\left(\mu_{t_{\mathrm{L}}}+\mu_{b_{\mathrm{L}}}-2 \mu_{t_{\mathrm{R}}}+2 \mu_{h}\right)+\Gamma_{h} \mu_{h},
\end{aligned}
$$

and $\delta \mathcal{C}_{2}^{i}=-\Gamma_{\text {tot }}^{i} u_{i}-v_{w} K_{0}^{i} \delta \overline{\mathcal{C}}_{1}^{i}$. Explicit equations for light quarks are not needed, even though their chemical potentials appear in the strong sphaleron rate $\tilde{\Gamma}_{\mathrm{SS}}\left[\mu_{i}\right]=$ $\Gamma_{\mathrm{SS}} \sum_{q}\left(\mu_{q_{\mathrm{L}}}-\mu_{q_{\mathrm{R}}}\right)$, since their chemical potentials can be

\footnotetext{
${ }^{7}$ We differ from the notation of FH06 by keeping track of the asymmetry in the right helicity sector with $t_{R}$ instead of its conjugate $t_{\mathrm{R}}^{c}$. The $C P$-odd asymmetries of the two species have a relative sign, $\mu_{t_{R}}=-\mu_{t_{\mathrm{R}}^{c}}$, since each $C P$-odd variable represents the difference between the two $C$-conjugate species. Likewise, the source for $\mu_{t_{R}}$ corresponds to $h=1$, which is opposite to that for $\mu_{t_{R}^{c}}$.
}

determined analytically. Light quarks are activated only by strong sphalerons, and in the approximation of no Yukawa mixing, $\mu_{q_{\mathrm{R}}}=-\mu_{q_{\mathrm{L}}}$ for all light species. Then using baryon number conservation (neglecting electroweak sphalerons, which are slow on the relevant time scale), $B=\sum_{q}\left(n_{q}-\bar{n}_{q}\right)=0$, one finds

$$
\mu_{q_{\mathrm{L}}}=-\mu_{q_{\mathrm{R}}}=D_{0}^{t} \mu_{t_{\mathrm{L}}}+D_{0}^{b} \mu_{b_{\mathrm{L}}}+D_{0}^{t} \mu_{t_{\mathrm{R}}},
$$

where $D_{0}=\left\langle f_{0 w}^{\prime}\right\rangle$ is a special case of the function (26) with $\ell=0$, identical to $K_{1}^{\mathrm{FH}}$ for all $v_{w}$, as can be shown by partial integration. Using (54) the strong sphaleron rate can be written as

$$
\begin{aligned}
\tilde{\Gamma}_{\mathrm{SS}}\left[\mu_{i}\right]= & \Gamma_{\mathrm{SS}}\left(\left(1+9 D_{0}^{t}\right) \mu_{t_{\mathrm{L}}}+\left(1+9 D_{0}^{b}\right) \mu_{b_{\mathrm{L}}}\right. \\
& \left.-\left(1-9 D_{0}^{t}\right) \mu_{t_{\mathrm{R}}}\right) .
\end{aligned}
$$

Inelastic collisions induce mixing between the particle species. Equations (52) are nevertheless linear in $\mu_{i}$ and $u_{i}$, and can be written in the compact matrix form

$$
A U^{\prime}-\Gamma U=S,
$$

where $U^{T} \equiv\left(w_{t_{\mathrm{L}}}^{T}, w_{b_{\mathrm{L}}}^{T}, w_{t_{\mathrm{R}}}^{T}, w_{h}^{T}\right), A=\operatorname{diag}\left(A_{t_{\mathrm{L}}}, A_{t_{\mathrm{R}}}, A_{b_{\mathrm{L}}}, A_{h}\right)$ is tridiagonal, and the matrix $\Gamma$ combines the $m_{i}^{2 \prime} B_{i}$ and collision terms. The source vector is $S^{T}=$ $\left(S_{t}^{T}, S_{b}^{T},-S_{t}^{T}, S_{h}^{T}\right)$ with $S_{i}$ defined in Eq. (52). Because of its block structure, $A$ is easily inverted to yield

$$
U^{\prime}=A^{-1} \Gamma U+A^{-1} S .
$$

This system is best solved using relaxation methods [50] since shooting tends to be unstable. The $8 \times 8$ matrix $A^{-1} \Gamma$ is the Jacobian of the differential equation network and its eigenvalues' signs distinguish the growing and decaying modes at the boundaries. This information may help to improve the numerical stability in more complicated systems.

Once the chemical potentials for the perturbations are determined, the baryon asymmetry follows from integrating them in the sphaleron rate equation. Following, e.g., Ref. [51] (but including the full Lorentz-covariant relations) we find ${ }^{8}$

$$
\eta_{B}=\frac{405 \Gamma_{\mathrm{sph}}}{4 \pi^{2} v_{w} \gamma_{w} g_{*} T} \int d z \mu_{B_{\mathrm{L}}} f_{\mathrm{sph}} e^{-45 \Gamma_{\mathrm{sph}}|z| / 4 v_{w} \gamma_{w}} .
$$

The seed asymmetry in Eq. (58) is the chemical potential for left-handed baryon number, $\mu_{B_{\mathrm{L}}}=\frac{1}{2} \sum_{q} \mu_{q_{\mathrm{L}}}$, which can be written in terms of $\mu_{t_{\mathrm{L}}}, \mu_{b_{\mathrm{L}}}$, and $\mu_{t_{\mathrm{R}}}$ using baryon number conservation:

\footnotetext{
${ }^{8}$ To our knowledge, the $1 / \gamma_{w}$ factor in front has been omitted in previous literature. It arises from the change of variable $d t \rightarrow$ $d z /\left(v_{w} \gamma_{w}\right)$ with $z$ in the rest frame of the wall.
} 
$\mu_{B_{\mathrm{L}}}=\frac{1}{2}\left(1+4 D_{0}^{t}\right) \mu_{t_{\mathrm{L}}}+\frac{1}{2}\left(1+4 D_{0}^{b}\right) \mu_{b_{\mathrm{L}}}+2 D_{0}^{t} \mu_{t_{\mathrm{R}}}$.

The function $f_{\mathrm{sph}}(z)=\min \left(1,2.4 \frac{\Gamma_{\mathrm{sph}}}{T} e^{-40 h(z) / T}\right)$ is designed to smoothly interpolate between the sphaleron rates in the broken and unbroken phases. $g_{*}$ is the number of degrees of freedom in the heat bath; in the standard model $g_{*}=106.75$.

\section{COMPARISON TO FHO6}

We can now compare our improved fluid equations (52) and (53) to those of FH06. The only difference between the two lies in the definition of the various coefficient functions, which we have renamed at the same time correcting and generalizing them to arbitrary wall velocities. To facilitate the comparison the results are collected in a dictionary translating between the two naming schemes in Table I. Our equations agree with those of FH06, when one assumes $h \rightarrow-1$ and $s_{\mathrm{p}} \rightarrow \operatorname{sign}\left(p_{z}\right)$ in the sources and replaces the coefficent functions according to Table I.

For the interaction rates we use the values given in [47]: $\Gamma_{\mathrm{sph}}=1.0 \times 10^{-6} \mathrm{~T}, \Gamma_{\mathrm{SS}}=4.9 \times 10^{-4} \mathrm{~T}, \Gamma_{y}=4.2 \times 10^{-3} \mathrm{~T}$, $\Gamma_{m}=m_{t}^{2} /(63 \mathrm{~T})$, and $\Gamma_{h}=m_{W}^{2} /(50 \mathrm{~T})$, where the top mass is as given in (48) and $m_{W}^{2} \equiv g^{2} h(z)^{2} / 4$. Furthermore the total interaction rates were defined as $\Gamma_{\text {tot }}^{i}=K_{4, i}^{\mathrm{FH}} /$ $\left(D_{i} K_{1, i}^{\mathrm{FH}}\right)$ with a quark diffusion constant $D_{q}=6 / T$ and a Higgs diffusion constant $D_{h}=20 / T$. The numerical impact of the Higgs and bottom masses is found to be quite small, and following FH06 we take them to be

TABLE I. A dictionary between the CK (this work) and the FH06 functions, depending upon $x=m / T$ and wall velocity $v_{w}$. They generally differ from each other at large $v_{w}$. Functions that are equivalent are marked by an equality sign in the middle column. The double exclamation mark indicates functions that do not agree even for small $v_{w}$, and single exclamation marks signal the source terms omitted in FH06.

\begin{tabular}{|c|c|c|}
\hline CK & & FH06 \\
\hline$D_{0}(x)$ & $=$ & $K_{1}^{\mathrm{FH}}(x)$ \\
\hline$D_{1}\left(x, v_{w}\right)$ & $=$ & $-v_{w} K_{1}^{\mathrm{FH}}(x)$ \\
\hline$D_{2}\left(x, v_{w}\right)$ & & $K_{4}^{\mathrm{FH}}(x)$ \\
\hline$Q_{1}\left(x, v_{w}\right)$ & & $K_{2}^{\mathrm{FH}}(x)$ \\
\hline$Q_{2}\left(x, v_{w}\right)$ & $!$ & 0 \\
\hline$R=-v_{w}$ & $=$ & $v_{w} \tilde{K}_{5}^{\mathrm{FH}}$ \\
\hline $\bar{R}\left(x, v_{w}\right)$ & !! & $\tilde{K}_{6}^{\mathrm{FH}}(x)$ \\
\hline$Q_{1}^{e}\left(x, v_{w}\right)$ & $!$ & 0 \\
\hline$Q_{2}^{e}\left(x, v_{w}\right)$ & & $K_{3}^{\mathrm{FH}}(x)$ \\
\hline$Q_{1}^{8 o}\left(x, v_{w}\right)$ & ! & 0 \\
\hline$Q_{2}^{8 o}\left(x, v_{w}\right)$ & & $K_{8}^{\mathrm{FH}}(x)$ \\
\hline$Q_{1}^{9 o}\left(x, v_{w}\right)$ & $!$ & 0 \\
\hline$Q_{2}^{9 o}\left(x, v_{w}\right)$ & & $K_{9}^{\mathrm{FH}}(x)$ \\
\hline$K_{0}(x)$ & !! & 1 \\
\hline
\end{tabular}
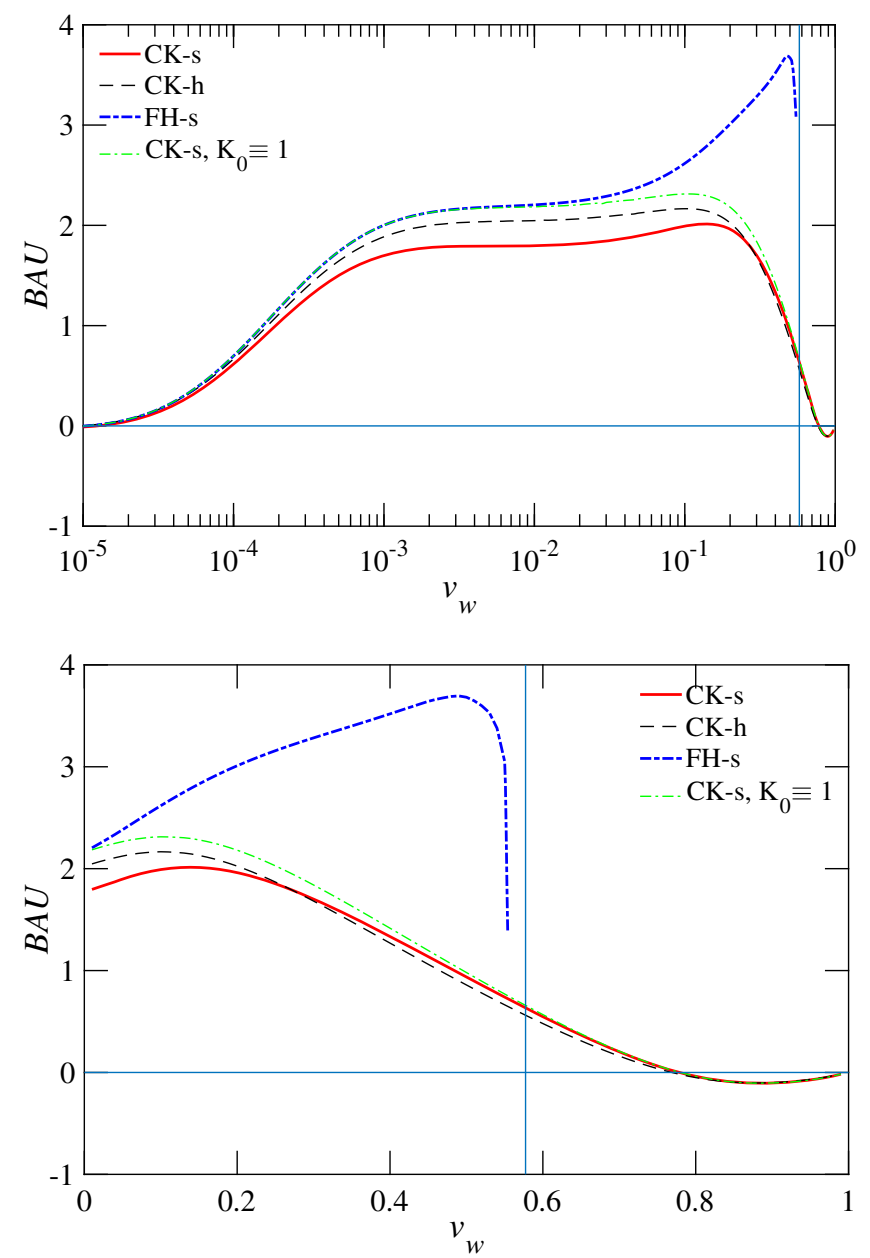

FIG. 3. Predicted baryon asymmetry in units of observed asymmetry for the fiducial profile as a function of the wall velocity $v_{w}$. From the logarithmic scale plot (upper panel) one can appreciate the good agreement at small $v_{w}$. Note the vanishing of BAU for $v_{w} \lesssim 10^{-5}$ due to the onset of thermal equilibrium. The linear scale (lower panel) expands the large $v_{w}$ region more relevant for strong transitions. The thin vertical line shows the sound speed $v_{s}=1 / \sqrt{3}$.

massless. Many of these rates have been quite roughly estimated, in some cases going back to the early reference [49], and deserve to be updated. We hope to make better determinations in an upcoming paper.

We display dependences of the predicted baryon asymmetry of the universe (BAU) normalized to the observed value, $\mathrm{BAU} \equiv \eta_{B} / \eta_{B \text {,obs }}$, in Fig. 3 . In both panels the thick red solid lines labeled "CK-s" correspond to the improved fluid equations with the spin- $s$ source, where we set $s_{\mathrm{p}} \rightarrow$ $\operatorname{sign}\left(p_{z}\right)$ and $h=-1$ in Eqs. (39)-(41). The thick dashdotted blue lines labeled "FH-s" correspond to the same spin- $s$ source, but using the FH06 equations. ${ }^{9}$ Thin dashed

\footnotetext{
${ }^{9}$ We switched for the sign of the source in FH06, however, so that the sign of the BAU matches in both cases.
} 


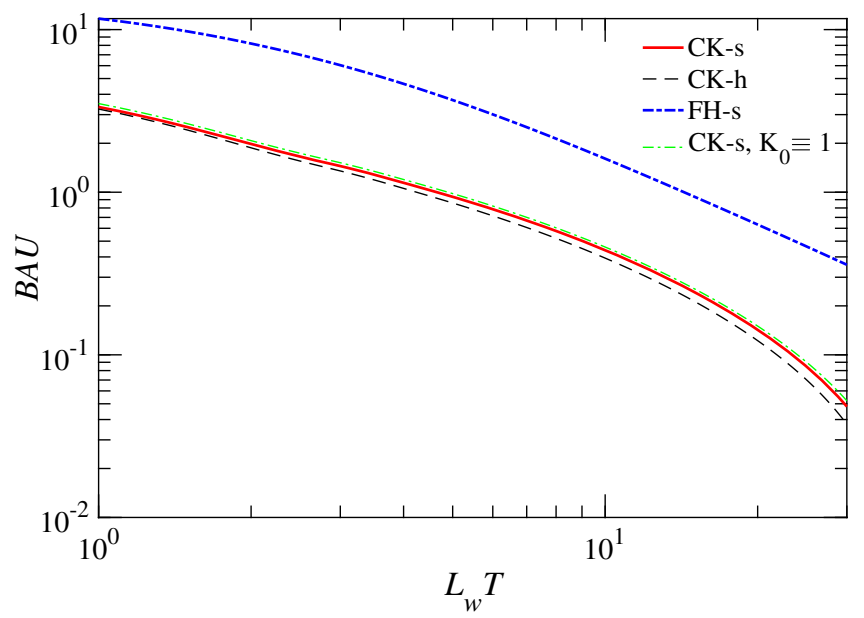

FIG. 4. Predicted baryon asymmetry (in units of observed asymmetry for the fiducial profile. as a function of the Higgs wall width $L_{w}$ for fixed $v_{w}=0.5$.

green lines labeled "CK- $s, K_{0}=0$ " correspond to the case where we set the $K_{0}$-function to unity in the otherwise accurate equations. The thin black dashed lines labeled "CK- $h$ " correspond to the improved fluid equations with the helicity source, still taking $h=-1$, but with $s_{\mathrm{p}}$ given by Eq. (5).

Clearly all approximations agree very well for $v_{w} \ll 1$ as expected, since the two sets of functions largely agree in the small $v_{w}$-limit; for $v_{w} \lesssim 0.01$, the only significant difference between the CK and the FH06 solutions comes from $K_{0}$. For larger $v_{w}$ the predictions differ significantly, in accord with our general arguments. In particular, the FH prediction plummets as $v_{w}$ approaches the sound speed $v_{s}=1 / \sqrt{3}$, shown by the thin vertical line in the plots. The more exact treatment, on the other hand, does nothing special near $v_{w}=v_{s}$; as expected the BAU smoothly decays as $v_{w} \rightarrow 1$.

Using the spin- $s$ source corresponds to identifying chirality with the eigenstates of spin in the z-direction, in the frame where the parallel momentum of the state vanishes, whereas the helicity source identifies chirality with helicity. The difference between the two is found to be small, due to the two bases becoming degenerate in the massless limit; in our example all fermions are massless in front of the wall.

In Fig. 4 we show the dependence on $L_{w}$ with $v_{w}=$ 0.5 held fixed. The FH prediction is substantially higher than the accurate value, and its ratio to the correct solution remains nearly constant. To summarize, our results and those of $\mathrm{FH}$ agree reasonably well for small $v_{w}$, but the improved fluid equations should be used for $v_{w} \gtrsim 0.1$ to get accurate results, and for $v_{w} \gtrsim v_{s}$ they are essential, since the $\mathrm{FH}$ equations incorrectly predict a vanishing BAU.

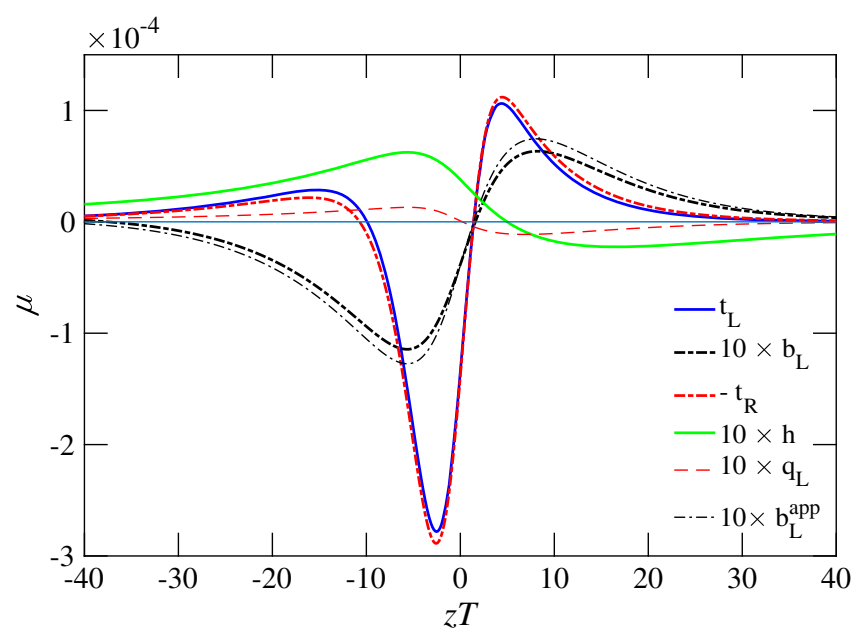

FIG. 5. Chemical potentials from the improved fluid equations for the fiducial case and for the spin- $s$ source. In addition to the chemical potentials in the network, we show light quark chemical potential $\mu_{q}$ corresponding to Eq. (54) and the approximation $\mu_{b_{\mathrm{L}}^{\text {app }}} \equiv-\left(D_{0}^{t} / D_{0}^{b}\right)\left(\mu_{t_{\mathrm{L}}}+\mu_{t_{\mathrm{R}}}\right)$.

\section{COMPARISON TO OTHER FORMALISMS}

There has been a long-standing divide among practitioners of electroweak baryogenesis as to which transport equations to use; yet no systematic comparison between them has been made in the literature. We undertake to do so in this section, continuing with the ansatz for the wall profiles and spatially varying top quark mass (48) and (50) introduced previously.

The VEV-insertion formalism is derived at the level of the integrated particle densities, which is equivalent to the formalism introduced in Refs. [49,52], consisting of coupled second-order diffusion equations for the local particle densities, in matrix form,

$$
D \mu^{\prime \prime}+v_{w} \mu^{\prime}-\delta C[\mu]=S .
$$

Here $\mu=\left(\mu_{t_{\mathrm{L}}}, \mu_{b_{\mathrm{L}}}, \mu_{t_{\mathrm{R}}}, \mu_{h}\right)^{T}$ and $S=\left(S_{t}, 0,-S_{t}, 0\right)^{T}, D=$ $\operatorname{diag}\left(D_{q}, D_{q}, D_{q}, D_{h}\right)$ is a diagonal matrix of diffusion coefficients, and $\delta C[\mu]$ is the inelastic collision integrals. The unsourced equation for $\mu_{b_{\mathrm{L}}}$ is usually omitted in the literature $[53,54]$. In this case baryon number conservation (as discussed above) leads to conditions $\mu_{q_{\mathrm{L}}}=\mu_{q_{\mathrm{R}}}$ and $\mu_{b_{\mathrm{L}}}=-\left(D_{0}^{t} / D_{0}^{b}\right)\left(\mu_{t_{\mathrm{L}}}+\mu_{t_{\mathrm{R}}}\right)[49,53]$. In fact, this is a reasonable approximation, as one can see from Fig. 5, where we plot the chemical potentials for our fiducial case (51) and for the spin- $h$ source using our improved fluid equations. For the purpose of comparing the formalisms, however, we have included the $b_{L}$ degrees of freedom in the diffusion equation networks.

In order to compare the system (60) to our improved equations (52), we need to find the equivalent source term 
to use in (60). The standard way to do this [29] is by eliminating $u$ from the WKB equations (35), neglecting all $\left(m^{2}\right)^{\prime} \mu$ and $\left(m^{2}\right)^{\prime} u$ terms in the derivation. It is easy to show that this procedure yields the following results adequate for the WKB picture, ${ }^{10,11}$

$$
\begin{aligned}
D_{\mathrm{WKB}} & =\frac{D_{2}-v_{w}^{2} D_{0}}{D_{0} \Gamma_{\mathrm{tot}}}, \\
S_{\mathrm{WKB}} & =\frac{S_{1}}{D_{0}}-\frac{v_{w} S_{1}^{\prime}+S_{2}^{\prime}}{D_{0} \Gamma_{\mathrm{tot}}}, \\
\delta C_{\mathrm{WKB}} & =\frac{K_{0}}{D_{0}} \delta \overline{\mathcal{C}}[\mu],
\end{aligned}
$$

where $\delta \overline{\mathcal{C}}[\mu]$ terms are given in (53). In the small $v_{w}$-limit $D_{\mathrm{WKB}}^{\alpha} \rightarrow D_{\alpha}$ and $S_{\mathrm{WKB}}^{\alpha} \rightarrow D_{\alpha} S_{\alpha, 2}^{\prime} /\left\langle v_{z}^{2}\right\rangle$ for each species $\alpha=q, h$, in agreement with Ref. [29].

The VEV-insertion formalism predicts a very different form for the source term in (60). We give a detailed derivation in Appendix B. The result, normalized as in Eq. (60), is

$$
S_{\mathrm{VEV}}^{t}=v_{w} \frac{N_{c} I}{2 \pi^{2} D_{0} T} m_{t}^{2}(z) \theta^{\prime}(z)
$$

where $N_{c}=3$ is the number of colors of the top quark and $I \cong 0.4$ is an integral given in Appendix B.

As for the diffusion term, no dependence of $D$ on $v_{w}$ is considered in the earlier literature in the VEV insertion approach. Accordingly, we will use

$$
D_{\mathrm{VEV}}^{\alpha}=D_{\alpha} .
$$

As mentioned above, we employ the same equation network for the WKB- and the VEV-insertion mechanisms, so that slightly upgrading the network of Ref. [54] to include $b_{L}$, we set

$$
\delta C_{\mathrm{VEV}}=\delta \overline{\mathcal{C}}[\mu],
$$

where as before $\delta \overline{\mathcal{C}}[\mu]$ corresponds to Eq. (53).

We can now compare the semiclassical and VEVinsertion formalism predictions for the BAU on a level playing ground, using the diffusion equations (60). The

\footnotetext{
${ }^{10}$ One should not confuse the diffusion coefficients with the fluid equation coefficient functions $D_{i}$. The latter are distinguished from the former by the fact that they are always associated with a numeral index.

${ }^{11}$ Note that the numerator in the diffusion coefficient $D_{\mathrm{WKB}}$ is just the determinant of the matrix $A$ in fluid equations (35). Thus, the critical speed condition discussed in Sec. III F corresponds to zero diffusion length. For $v_{w}>v_{c}$ the diffusion length would be negative, which is, of course, unphysical. This is why the FH06 solutions go to zero as $v_{w} \rightarrow v_{c}$. However, in the improved equations $D_{\mathrm{WKB}}$ and hence the diffusion length remain positive until $v_{w}=1$.
}
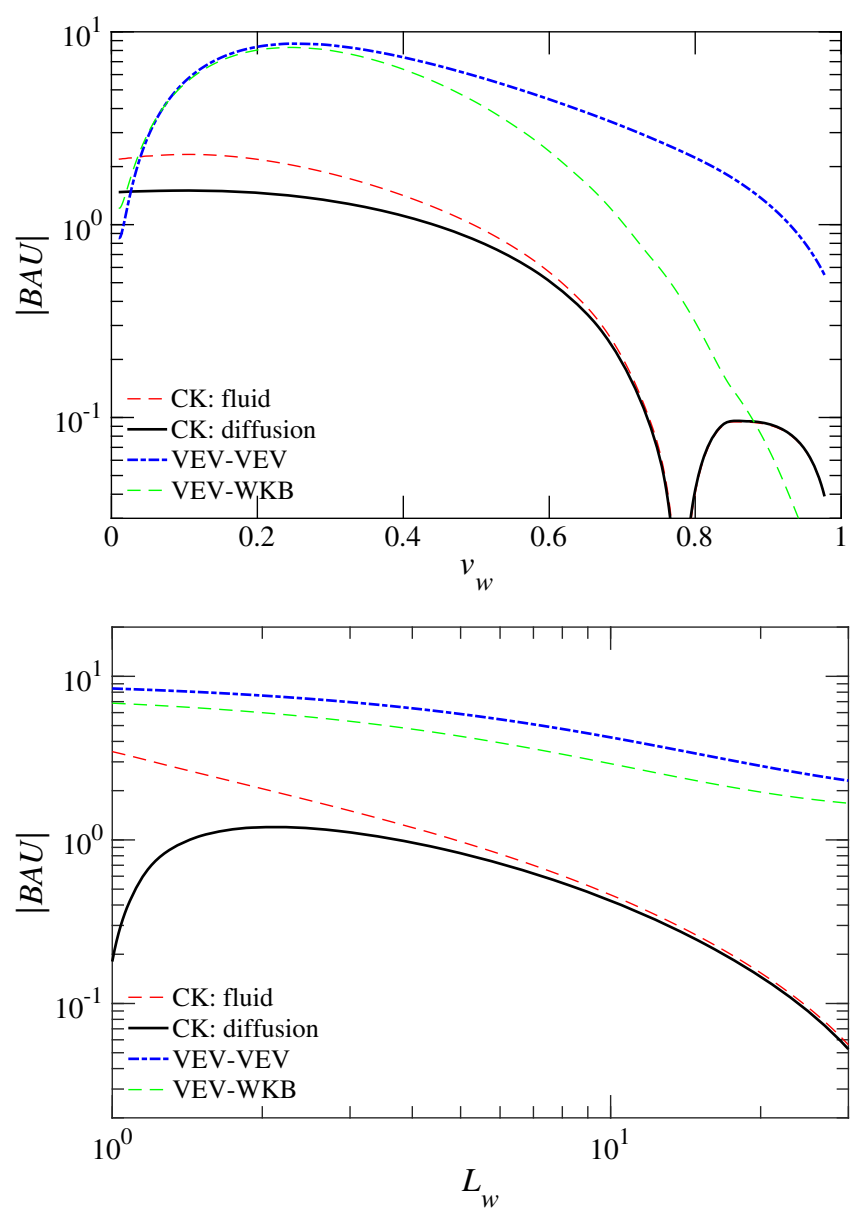

FIG. 6. Predicted absolute value of the baryon asymmetry (in units of observed asymmetry) for the fiducial model as a function of wall velocity $v_{w}$ (top) and Higgs wall width $L_{w}$ (bottom). "CK-fluid" denotes the two-moment result derived previously; "CK-diffusion" is the diffusion equation approximation (60) to the two-moment network; "VEV-VEV" is the result from the full VEV-insertion formalism; "VEV-WKB" is a hybrid result where the diffusion and collision terms are the same as in the semiclassical (CK) formalism, and only the VEV-insertion source term is different.

results are shown in Fig. 6. The upper panel displays the absolute value of the BAU $\equiv\left|\eta_{B} / \eta_{B \text {,obs }}\right|$ as a function of the wall velocity for our fiducial model (51) and for the spin- $h$ source. The thick solid black line corresponds to the solution of the diffusion equation (60) with the WKB variables (61). The dashed red line shows for comparison the result of our earlier calculation with improved fluid equations. The two semiclassical approximations agree remarkably well, in particular for large $v_{w}$.

Considering the VEV-insertion formalism, the thick dash-dotted blue line (labeled "VEV-VEV") displays the result of using the parameters (62)-(64) in Eq. (60). The VEV-insertion method predicts 10-50 times larger asymmetry than does the semiclassical method. The thin dashed green line (labeled "VEV-WKB") shows the result of using 
the VEV-insertion source, but the WKB diffusion constant and collision terms from Eq. (61). For small $v_{w}$ the difference between the VEV-insertion method and the WKB method is dominated by the source terms. However, for $v_{w} \gtrsim 0.4$ a further deviation is caused by the $v_{w}$-dependence of the evolution equations.

The lower panel displays the dependence of BAU on the wall width $L_{w}$ for $v_{w}=0.5$. For $L_{w} T \gtrsim 2$ the two semiclassical approximations agree very well. The difference at small $L_{w}$ is expected, because the diffusion approximation neglects several $\mathrm{m}^{2 \prime}$ corrections included in the fluid equations. As the wall width increases, the semiclassical BAU decreases rapidly. This is the expected behavior as the system becomes increasingly classical. The VEV-insertion predicts a much slower decrease of BAU with $L_{w}$.

In our opinion, the semiclassical formalism is the more reliable one. First, it has a clearly established small expansion parameter: $\left(L_{w} T\right)^{-1}$, and the results show the expected behavior as a function of this parameter. Second, it has been derived both using WKB methods $[29,37,38]$ and from the fundamental CTP formalism [30-33]. Third, the semiclassical limit has been recently shown to arise in a full quantum mechanical treatment without any gradient expansion [55] (for earlier related work see [56] and [57-63]).

In the VEV-insertion approach the expansion parameter is not as clearly defined: in addition to expanding in powers of $m(z) / T$, there is also a gradient expansion, which, however, always results in fewer derivatives acting on the source term than in the semiclassical approach, leading to the different dependence on the wall width shown in Fig. 6. Moreover, the source term is infrared singular, cured only by introducing a damping term that is not related to the quantum physics of the problem. This can be seen in Eq. (B1), which blows up when the damping rate $\gamma \rightarrow 0$. The lack of convergence of the expansion in $m / T$ was recently investigated in Ref. [64], where it was shown that higher order terms are small only if $m / T<\sqrt{\alpha}$, where $\alpha$ is the relevant interaction strength of the fermion of mass $m$. There is a claim in the literature that the $m / T$ expansion can be avoided [65], but this is based upon a phenomenological approach which, although superficially similar to the VEV-insertion formalism, does not derive the transport equations from first principles within the CTP formalism.

\section{CONCLUSIONS}

In this paper we generalized the semiclassical fluid equations for electroweak baryogenesis to the regime of arbitrarily large wall velocities, showing that diffusion remains relatively efficient for $v_{w}$ exceeding the sound speed in the plasma. As a result, EWBG can be effective even for very strong transitions corresponding to detonations. We performed a detailed comparison between a new improved network of fluid equations and the previous formulation by Fromme and Huber [34]. For small wall velocities $v_{w} \lesssim 0.1$ the two formulations agree reasonably well, but for larger values, in particular for $v_{w}$ exceeding the sound speed, the improved formalism is indispensable.

We then quantitatively compared the semiclassical fluid equations to a competing framework, the VEV insertion method. To do so required reducing the semiclassical fluid equations, consisting of coupled equations for chemical potentials and velocity perturbations, to a set of (WKB) diffusion equations for the particle densities alone (while retaining the full velocity dependence). This was necessary because the VEV-insertion formalism is derived at the level of particle densities, and does not lend itself to a more accurate approximation of the Boltzmann equation in terms of coupled moments (including the velocity perturbation).

The WKB diffusion equations agree very well with the improved fluid equations in the semiclassical picture. The VEV insertion method, on the other hand, predicts the BAU is a factor of 10-50 times larger than in the semiclassical method. This difference arises mostly (especially for small $v_{w}$ ) from the different source terms in the semiclassical and the VEV-insertion schemes. We argued that the semiclassical results are more reliable, as they have been derived and verified in various different approaches and they, unlike the VEV-insertion results, show the expected parametric behaviors.

Although we have restricted our discussion to the $\mathrm{CP}$ odd perturbations in the plasma densities that govern the baryon asymmetry, analogous diffusion equations exist for the $C P$-even perturbations that determine the dynamics of the bubble wall and ultimately its friction and terminal speed [24]. The issues we have discussed here are also relevant for those equations, which were analyzed more recently by Ref. [66]. Although the authors of that paper avoided making any small- $v_{w}$ approximations, their conclusions concerning the limiting speed of diffusion differ from ours; they obtain the speed of sound rather than that of light. We can trace this difference to the fact that they use a specific ansatz for the form of the velocity perturbation $\delta f$ in Eq. (9), while we avoid such a choice, instead determining moments of $\delta f$ using the rules (28) and (29). ${ }^{12}$

Finally, we caution that while our improved fluid equations cure the incorrect dependence predicted by the FH06 network at large $v_{w}$, they still correspond to a loworder expansion in moments of the particle distribution functions $f$. One might reasonably expect that perturbations $\delta f$ could be highly non-Gaussian, such that going beyond second order in the moment expansion could significantly modify the results presented here. This is a question that deserves further study.

\footnotetext{
${ }^{12}$ In fact, during the course of this work, we studied a number of possible ansätze for $\delta f$ before abandoning this approach, because they all tend to predict critical speeds below $v_{w}=1$, contrary to the physical arguments that make us confident in this value.
} 


\section{ACKNOWLEDGMENTS}

We thank V. Cirigliano, C. Lee, T. Konstandin, M. Postma, and S. Tulin for useful correspondence. This work was supported by the Academy of Finland Grant No. 318319 and NSERC (Natural Sciences and Engineering Research Council, Canada).

\section{APPENDIX A: EXPLICIT FORMS FOR COEFFICIENT FUNCTIONS}

All coefficient functions are expressed as integrals over the distribution functions in the wall rest frame that in general depend upon the local particle masses $m(z) / T$ and $v_{w}$, and they can be fermionic or bosonic even though the normalization factors $N_{i}$ are taken to refer to massless fermions. When evaluating these functions it is convenient to Lorentz transform the integration variables,

$$
\begin{gathered}
E=\gamma_{w}\left(E_{v}-v_{w} p_{z v}\right), \\
p_{z}=\gamma_{w}\left(p_{z v}-v_{w} E_{v}\right),
\end{gathered}
$$

where $E_{v}$ and $p_{x v}$ are the variables in the plasma frame. One can then use the fact that $\mathrm{d}^{3} p / E$ is invariant and $\gamma_{w}\left(E+v_{w} p_{z}\right) \rightarrow E_{v}$ so that $f_{0 w} \rightarrow f_{0}$ (and similarly for the derivatives of $\left.f_{0 w}\right)$. All coefficient functions can then be written as a two-dimensional integral of a generic form

$$
\left\langle\frac{p_{z}^{n}}{E^{m}} V \mathcal{F}_{0 w}\right\rangle=T^{n-m-k} K\left(\mathcal{F}_{0} ; V ; n, m\right),
$$

where $k=0$ for $\mathcal{F}_{0 w}=f_{0 w}, k=1$ for $\mathcal{F}_{0 w}=f_{0 w}^{\prime}$ and $k=2$ for $\mathcal{F}_{0 w}=f_{0 w}^{\prime \prime}$ and the dimensionless integral

$$
\begin{aligned}
K\left(\mathcal{F}_{0} ; V ; n, m\right) \equiv & -\frac{3}{\pi^{2} \gamma_{w}} \int_{x}^{\infty} \mathrm{d} w \int_{-1}^{1} \mathrm{~d} y \\
& \times \frac{\tilde{p}_{w} \tilde{p}_{z}^{n}}{\tilde{E}^{m-1}} V\left(w, y, v_{w}, x\right) \mathcal{F}_{0}(w),
\end{aligned}
$$

where $\tilde{p}_{w} \equiv \sqrt{w^{2}-x^{2}}, \quad \tilde{p}_{z}=\gamma_{w}\left(y \tilde{p}_{w}-w v_{w}\right)$, and $\tilde{E}=$ $\gamma_{w}\left(w-v_{w} y \tilde{p}_{w}\right)$. For $D_{\ell}, Q_{\ell}, Q_{\ell}^{e}$ and for $K_{1}$ the auxiliary function $V=1$, while for the $C P$-odd source functions $Q_{\ell}^{8 o}$ and $Q_{\ell}^{9 o}$ a more complicated structure $V=s_{\mathrm{p}} p_{z} / E_{z}$ appears. For the spin $s$ eigenstates this means

$$
V=V_{s}=\frac{\left|p_{z}\right|}{E_{z}}=\frac{\left|\tilde{p}_{z}\right|}{\sqrt{\tilde{p}_{z}^{2}+x^{2}}}
$$

and for helicity eigenstates

$$
V=V_{h} \equiv V_{s}^{2}\left(1-\frac{\tilde{p}_{z}^{2}}{\tilde{E}^{2}}\right)^{-1 / 2}
$$

where $\tilde{p}_{z}$ and $\tilde{E}$ are as given below (A3). Explicitly then

$$
\begin{aligned}
T D_{\ell} & =K\left(f_{0}^{\prime} ; 1 ; \ell, \ell\right), \\
T^{3} Q_{\ell} & =K\left(f_{0}^{\prime \prime} ; 1 ; \ell-1, \ell\right), \\
T^{2} Q_{\ell}^{e} & =K\left(f_{0}^{\prime} ; 1 ; \ell-1, \ell\right), \\
T^{3} Q_{\ell}^{8 o}= & \frac{1}{2} K\left(f_{0}^{\prime} ; V_{x} ; \ell-2, \ell\right), \\
T^{5} Q_{\ell}^{9 o}= & \frac{1}{4}\left[K\left(f_{0}^{\prime} ; V_{x} ; \ell-2, \ell+2\right),\right. \\
& \left.-\gamma_{w} K\left(f_{0}^{\prime \prime} ; V_{x} ; \ell-2, \ell+1\right)\right] .
\end{aligned}
$$

In particular, one can show that some of the coefficient functions are independent of the wall velocity or that their $v_{w}$-dependence factorizes simply

$$
\begin{aligned}
D_{0}(x) & =\frac{1}{\hat{N}_{1}} \int \mathrm{d}^{3} p_{v} f_{0}^{\prime}, \\
D_{1}\left(x, v_{w}\right) & =-v_{w} D_{0}(x), \\
Q_{1}\left(x, v_{w}\right) & =\frac{1}{\gamma_{w} \hat{N}_{1}} \int \frac{\mathrm{d}^{3} p_{v}}{2 E_{v}} f_{0}^{\prime \prime}, \\
Q_{1}^{e}\left(x, v_{w}\right) & =\frac{1}{\gamma_{w} \hat{N}_{1}} \int \frac{\mathrm{d}^{3} p_{v}}{2 E_{v}} f_{0}^{\prime}, \\
K_{0}(x) & =\frac{1}{\hat{N}_{1}} \int \mathrm{d}^{3} p_{v} f_{0} .
\end{aligned}
$$

All these integrals can easily be reduced to onedimensional integrals over $E_{v}$. The function $\bar{R}$ is a special case, whose one-dimensional integral representation was already given in (32).

\section{APPENDIX B: VEV-INSERTION SOURCE}

The exact form of the VEV-insertion source has never been derived for the model of $C P$-violation (48) adopted in this work, but similar expressions have been worked out for two-Higgs doublet models (2HDMs) where an analogous source is present. In $2 \mathrm{HDMs}$, there is an extra suppression factor $\sin ^{2}(2 \beta)$ where $\tan \beta=H_{2} / H_{1}$ that is not present in our model. The source term is therefore similar to Eq. (34) of Ref. [67], except for some typos and an error in that equation [68]. The correct expression is

$$
\begin{aligned}
S_{\mathrm{VEV}}= & \frac{v_{w} N_{c} m_{t}(z)^{2} \theta^{\prime}}{2 \pi^{2}} \int \mathrm{d} k k^{2} \\
& \times \operatorname{Im}\left[Z_{t_{L}}^{p} Z_{t_{R}}^{h} \frac{n_{F}\left(\mathcal{E}_{t_{R}}^{h *}\right)-n_{F}\left(\mathcal{E}_{t_{L}}^{p}\right)}{\left(\mathcal{E}_{t_{L}}^{p}-\mathcal{E}_{t_{R}}^{h *}\right)^{2}}\right. \\
& \left.+Z_{t_{L}}^{p} Z_{t_{R}}^{p} \frac{0+n_{F}\left(\mathcal{E}_{t_{R}}^{p}\right)+n_{F}\left(\mathcal{E}_{t_{L}}^{p}\right)}{\left(\mathcal{E}_{t_{L}}^{p}+\mathcal{E}_{t_{R}}^{p}\right)^{2}}+(p \leftrightarrow h)\right],
\end{aligned}
$$

where the superscripts $p, h$ refer to quasiparticle and hole excitations in the hot plasma, taken to be in the electroweak symmetric phase, and $n_{F}$ denotes the Fermi-Dirac 
distribution function evaluated at a complex energy $\mathcal{E}=E+i \gamma$, where $\gamma$ is the damping rate (thermal width) of the left- or right-handed top quark.

The error in the original expression for (B1) was that the term " 0 " was originally " 1 ," which leads to a UV-divergent integral. It is argued [69] that normal ordering removes this term, although no derivation has ever been published. The correction to a similar source term was mentioned in Ref. [70] (see [31] of that paper).

The VEV-insertion source has the peculiar property of being singular if the energies $\mathcal{E}$ are real. The regulating damping rate is dominated by the QCD contribution [71]:

$$
\gamma=\frac{5.7 g_{s}^{2}}{12 \pi} T
$$

The real parts of the energies are given approximately by

$$
\begin{aligned}
E^{p} & =k^{2}+\frac{m^{2}}{m+k}, \\
E^{h} & =k\left(1-0.45 e^{-1.5(k / m)^{2}}\right)
\end{aligned}
$$

(these are good analytic fits to the numerical solutions for the poles of the thermally corrected propagators) with $m$ being the thermal mass for the chirality of interest [72],

$$
\begin{aligned}
& m_{L}^{2}=\left(g_{s}^{2} / 6+3 g_{2}^{2} / 32+y_{t}^{2} / 16\right) T^{2}, \\
& m_{R}^{2}=\left(g_{s}^{2} / 6+y_{t}^{2} / 8\right) T^{2} .
\end{aligned}
$$

The wave-function normalization factors are given by

$$
Z=\frac{E^{2}-k^{2}}{m^{2}}
$$

for each kind of particle or hole. Taking the known values of the coupling constants, the integral in (B1) then becomes $I \cong 0.40 T$. It is normalized in this form to be a source for the diffusion equation for particle densities. To convert it to a source for the top quark chemical potential, we use the relation $\delta n=N_{c} g_{t} D_{0} T^{2} \mu / 6$ for a chiral quark with $g_{t}=2$ spin degrees of freedom. Hence $S_{t}=S_{\mathrm{VEV}} /\left(D_{0} T^{2}\right)$ to obtain Eq. (62).

The same source term has been derived recently in Ref. [64] [see Eq. (55)], in a simpler form where no distinction is made between particle and hole states, and the quasiparticle dispersion relations are approximated by the usual relativistic ones. We find that the numerical difference is slight, giving $I \cong 0.37 T$ rather than $0.40 T$.
[1] K. Kajantie, M. Laine, K. Rummukainen, and M.E. Shaposhnikov, Is There a Hot Electroweak Phase Transition at $m_{H} \gtrsim m_{W}$ ?, Phys. Rev. Lett. 77, 2887 (1996).

[2] A. I. Bochkarev, S. V. Kuzmin, and M. E. Shaposhnikov, Electroweak baryogenesis and the Higgs boson mass problem, Phys. Lett. B 244, 275 (1990).

[3] A. G. Cohen, D. B. Kaplan, and A. E. Nelson, Weak scale baryogenesis, Phys. Lett. B 245, 561 (1990).

[4] A. G. Cohen, D. B. Kaplan, and A. E. Nelson, Baryogenesis at the weak phase transition, Nucl. Phys. B349, 727 (1991).

[5] N. Turok and J. Zadrozny, Electroweak baryogenesis in the two doublet model, Nucl. Phys. B358, 471 (1991).

[6] V. Vaskonen, Electroweak baryogenesis and gravitational waves from a real scalar singlet, Phys. Rev. D 95, 123515 (2017).

[7] G. C. Dorsch, S. J. Huber, T. Konstandin, and J. M. No, A second higgs doublet in the early universe: Baryogenesis and gravitational waves, J. Cosmol. Astropart. Phys. 05 (2017) 052.

[8] L. Bian, H.-K. Guo, and J. Shu, Gravitational Waves, baryon asymmetry of the universe and electric dipole moment in the $C P$-violating NMSSM, Chin. Phys. C 42, 093106 (2018).
[9] S. Akula, C. Balázs, L. Dunn, and G. White, Electroweak baryogenesis in the $\mathbb{Z}_{3}$-invariant NMSSM, J. High Energy Phys. 11 (2017) 051.

[10] F. P. Huang, Z. Qian, and M. Zhang, Exploring dynamical $C P$ violation induced baryogenesis by gravitational waves and colliders, Phys. Rev. D 98, 015014 (2018).

[11] B. Grzadkowski and D. Huang, Spontaneous $C P$-violating electroweak baryogenesis and dark matter from a complex singlet scalar, J. High Energy Phys. 08 (2018) 135.

[12] P. Huang, A. J. Long, and L.-T. Wang, Probing the electroweak phase transition with higgs factories and gravitational waves, Phys. Rev. D 94, 075008 (2016).

[13] M. Artymowski, M. Lewicki, and J. D. Wells, Gravitational wave and collider implications of electroweak baryogenesis aided by non-standard cosmology, J. High Energy Phys. 03 (2017) 066.

[14] K. Hashino, M. Kakizaki, S. Kanemura, P. Ko, and T. Matsui, Gravitational waves and Higgs boson couplings for exploring first order phase transition in the model with a singlet scalar field, Phys. Lett. B 766, 49 (2017).

[15] W. Chao, H.-K. Guo, and J. Shu, Gravitational wave signals of electroweak phase transition triggered by dark matter, J. Cosmol. Astropart. Phys. 09 (2017) 009.

[16] A. Beniwal, M. Lewicki, J. D. Wells, M. White, and A. G. Williams, Gravitational wave, collider and dark matter 
signals from a scalar singlet electroweak baryogenesis, J. High Energy Phys. 08 (2017) 108.

[17] G. Kurup and M. Perelstein, Dynamics of electroweak phase transition in singlet-scalar extension of the standard model, Phys. Rev. D 96, 015036 (2017).

[18] I. Baldes, Gravitational waves from the asymmetricdark-matter generating phase transition, J. Cosmol. Astropart. Phys. 05 (2017) 028.

[19] A. Angelescu and P. Huang, Multistep strongly first order phase transitions from new fermions at the TeV scale, Phys. Rev. D 99, 055023 (2019).

[20] A. Beniwal, M. Lewicki, M. White, and A. G. Williams, Gravitational waves and electroweak baryogenesis in a global study of the extended scalar singlet model, J. High Energy Phys. 02 (2019) 183.

[21] A. Ahriche, K. Hashino, S. Kanemura, and S. Nasri, Gravitational waves from phase transitions in models with charged singlets, Phys. Lett. B 789, 119 (2019).

[22] P. Athron, C. Balazs, A. Fowlie, G. Pozzo, G. White, and Y. Zhang, Strong first-order phase transitions in the NMSSM-a comprehensive survey, J. High Energy Phys. 11 (2019) 151.

[23] G. D. Moore and T. Prokopec, Bubble Wall Velocity in a First Order Electroweak Phase Transition, Phys. Rev. Lett. 75, 777 (1995).

[24] G. D. Moore and T. Prokopec, How fast can the wall move? A Study of the electroweak phase transition dynamics, Phys. Rev. D 52, 7182 (1995).

[25] G. D. Moore, Electroweak bubble wall friction: Analytic results, J. High Energy Phys. 03 (2000) 006.

[26] P. John and M. G. Schmidt, Do stops slow down electroweak bubble walls?, Nucl. Phys. B598, 291 (2001); Erratum, Nucl. Phys. B648, 449 (2003).

[27] J. R. Espinosa, T. Konstandin, and F. Riva, Strong electroweak phase transitions in the standard model with a singlet, Nucl. Phys. B854, 592 (2012).

[28] M. Joyce, T. Prokopec, and N. Turok, Nonlocal electroweak baryogenesis. Part 2: The Classical regime, Phys. Rev. D 53, 2958 (1996).

[29] J. M. Cline, M. Joyce, and K. Kainulainen, Supersymmetric electroweak baryogenesis, J. High Energy Phys. 07 (2000) 018.

[30] K. Kainulainen, T. Prokopec, M. G. Schmidt, and S. Weinstock, First principle derivation of semiclassical force for electroweak baryogenesis, J. High Energy Phys. 06 (2001) 031.

[31] K. Kainulainen, T. Prokopec, M. G. Schmidt, and S. Weinstock, Semiclassical force for electroweak baryogenesis: Three-dimensional derivation, Phys. Rev. D 66, 043502 (2002).

[32] T. Prokopec, M. G. Schmidt, and S. Weinstock, Transport equations for chiral fermions to order $\mathrm{h}$ bar and electroweak baryogenesis. Part 1, Ann. Phys. (Amsterdam) 314, 208 (2004).

[33] T. Prokopec, M. G. Schmidt, and S. Weinstock, Transport equations for chiral fermions to order h-bar and electroweak baryogenesis. Part II, Ann. Phys. (Amsterdam) 314, 267 (2004).

[34] L. Fromme and S. J. Huber, Top transport in electroweak baryogenesis, J. High Energy Phys. 03 (2007) 049.
[35] A. Riotto, Towards a nonequilibrium quantum field theory approach to electroweak baryogenesis, Phys. Rev. D 53, 5834 (1996).

[36] A. Riotto, Supersymmetric electroweak baryogenesis, nonequilibrium field theory and quantum Boltzmann equations, Nucl. Phys. B518, 339 (1998).

[37] J. M. Cline, M. Joyce, and K. Kainulainen, Supersymmetric electroweak baryogenesis in the WKB approximation, Phys. Lett. B 417, 79 (1998); Erratum, Phys. Lett. B 448, 321 (1999).

[38] J. M. Cline, M. Joyce, and K. Kainulainen, Erratum for 'Supersymmetric electroweak baryogenesis', arXiv:hep-ph/ 0110031 .

[39] T. Konstandin, T. Prokopec, and M. G. Schmidt, Kinetic description of fermion flavor mixing and $C P$-violating sources for baryogenesis, Nucl. Phys. B716, 373 (2005).

[40] T. Konstandin, T. Prokopec, M. G. Schmidt, and M. Seco, MSSM electroweak baryogenesis and flavor mixing in transport equations, Nucl. Phys. B738, 1 (2006).

[41] V. Cirigliano, C. Lee, M. J. Ramsey-Musolf, and S. Tulin, Flavored quantum boltzmann equations, Phys. Rev. D 81, 103503 (2010).

[42] V. Cirigliano, C. Lee, and S. Tulin, Resonant flavor oscillations in electroweak baryogenesis, Phys. Rev. D 84, 056006 (2011).

[43] J. M. Cline and K. Kainulainen, A New Source for Electroweak Baryogenesis in the MSSM, Phys. Rev. Lett. 85, 5519 (2000).

[44] M. Carena, M. Quiros, and C. E. M. Wagner, Opening the window for electroweak baryogenesis, Phys. Lett. B 380, 81 (1996).

[45] M. Carena, M. Quiros, A. Riotto, I. Vilja, and C. E. M. Wagner, Electroweak baryogenesis and low-energy supersymmetry, Nucl. Phys. B503, 387 (1997).

[46] J. M. Cline, K. Kainulainen, and D. Tucker-Smith, Electroweak baryogenesis from a dark sector, Phys. Rev. D 95, 115006 (2017).

[47] L. Fromme, S. J. Huber, and M. Seniuch, Baryogenesis in the two-Higgs doublet model, J. High Energy Phys. 11 (2006) 038.

[48] M. Joyce, T. Prokopec, and N. Turok, Nonlocal electroweak baryogenesis. Part 1: Thin wall regime, Phys. Rev. D 53, 2930 (1996).

[49] P. Huet and A. E. Nelson, Electroweak baryogenesis in supersymmetric models, Phys. Rev. D 53, 4578 (1996).

[50] J. Cash and M. Wright, A deferred correction method for nonlinear two-point boundary value problems: Implementation and numerical evaluation, SIAM J. Sci. Stat. Comput. 12, 971 (1991).

[51] J. M. Cline, K. Kainulainen, and M. Trott, Electroweak baryogenesis in two higgs doublet models and B meson anomalies, J. High Energy Phys. 11 (2011) 089.

[52] A. G. Cohen, D. B. Kaplan, and A. E. Nelson, Diffusion enhances spontaneous electroweak baryogenesis, Phys. Lett. B 336, 41 (1994).

[53] D. J. H. Chung, B. Garbrecht, M. J. Ramsey-Musolf, and S. Tulin, Lepton-mediated electroweak baryogenesis, Phys. Rev. D 81, 063506 (2010).

[54] S. Tulin and P. Winslow, Anomalous $B$ meson mixing and baryogenesis, Phys. Rev. D 84, 034013 (2011). 
[55] H. Jukkala, K. Kainulainen, and O. Koskivaara, Quantum transport and the phase space structure of the Wightman functions, J. High Energy Phys. 01 (2020) 012.

[56] T. Prokopec, M. G. Schmidt, and J. Weenink, Exact solution of the Dirac equation with $C P$ violation, Phys. Rev. D 87, 083508 (2013).

[57] C. Fidler, M. Herranen, K. Kainulainen, and P. M. Rahkila, Flavoured quantum Boltzmann equations from cQPA, J. High Energy Phys. 02 (2012) 065.

[58] M. Herranen, K. Kainulainen, and P. M. Rahkila, Towards a kinetic theory for fermions with quantum coherence, Nucl. Phys. B810, 389 (2009).

[59] M. Herranen, K. Kainulainen, and P. M. Rahkila, Quantum kinetic theory for fermions in temporally varying backgrounds, J. High Energy Phys. 09 (2008) 032.

[60] M. Herranen, K. Kainulainen, and P. M. Rahkila, Kinetic theory for scalar fields with nonlocal quantum coherence, J. High Energy Phys. 05 (2009) 119.

[61] M. Herranen, Quantum kinetic theory with nonlocal coherence, Ph.D. thesis, Jyvaskyla U., 2009.

[62] M. Herranen, K. Kainulainen, and P. M. Rahkila, Coherent quasiparticle approximation (cQPA) and nonlocal coherence, J. Phys. Conf. Ser. 220, 012007 (2010).

[63] M. Herranen, K. Kainulainen, and P. M. Rahkila, Coherent quantum Boltzmann equations from cQPA, J. High Energy Phys. 12 (2010) 072.
[64] M. Postma and J. Van De Vis, Source terms for electroweak baryogenesis in the vev-insertion approximation beyond leading order, J. High Energy Phys. 02 (2020) 090.

[65] M. Carena, J. M. Moreno, M. Quiros, M. Seco, and C. E. M. Wagner, Supersymmetric $C P$ violating currents and electroweak baryogenesis, Nucl. Phys. B599, 158 (2001).

[66] T. Konstandin, G. Nardini, and I. Rues, From Boltzmann equations to steady wall velocities, J. Cosmol. Astropart. Phys. 09 (2014) 028.

[67] K. Blum, C. Delaunay, M. Losada, Y. Nir, and S. Tulin, $C P$ violation beyond the MSSM: Baryogenesis and electric dipole moments, J. High Energy Phys. 05 (2010) 101.

[68] S. Tulin (private communication).

[69] S. Lee (private communication).

[70] T. Liu, M. J. Ramsey-Musolf, and J. Shu, Electroweak Beautygenesis: From $b \rightarrow s C P$-violation to the Cosmic Baryon Asymmetry, Phys. Rev. Lett. 108, 221301 (2012).

[71] E. Braaten and R. D. Pisarski, Calculation of the quark damping rate in hot QCD, Phys. Rev. D 46, 1829 (1992).

[72] H. A. Weldon, Effective fermion masses of order gT in high temperature gauge theories with exact chiral invariance, Phys. Rev. D 26, 2789 (1982). 